\title{
Internet through the exploitation of Digital Terrestrial TV Networks
}

\author{
S.Venkatesh \\ Department of Electronics and Communication Engineering, Pondicherry Engineering College, Puducherry, India
}

\begin{abstract}
The Internet is paramount of modern life. As technology advances, our demand for bigger, better and faster Internet connections. Current Digital TVs (DTVs) which is universally growing across the world have the ability to connect to Internet. So, Digital Terrestrial TV network that also carry Internet data is the objective of this paper. This is another feasible advantage that digital terrestrial TV can offer customers .It would be a user-friendly method just to plug the computers to set-top boxes to access Internet. Consumers can find it convenient to pay for TV content and Internet usage separately. With several technologies like ATSC, DMB, DVB-T2 and ISDB-T already in existence, it is from this background that a concept to improve bandwidth, compression, source coding and line coding is suggested for maximum network exploitation.
\end{abstract}

Keywords: Digital Terrestrial TV (DTT), Advance Television System Committee (ATSC), Digital Multimedia Broadcasting (DMB), Digital Video Broadcasting- Terrestrial 2 (DVB-T2), Integrated Services Digital Broadcasting (ISDB), Orthogonal Frequency Division Multiplexing (OFDM), Digital Subscriber Line (DSL), Inverse Fourier Fast Fourier Transform (IFFT), Single Carrier(SC) and Multi Carrier(MC).

\section{I.INTRODUCTION}

The Internet is at once a world-wide broadcasting capability, a mechanism for information dissemination, and a medium for collaboration and interaction between individuals and their computers without regard for geographic location. Digital subscriber line (DSL; originally digital subscriber loop) is a family of technologies that are used to provide internet access by transmitting digital data over telephone lines. Digital terrestrial television (DTTV or DTT) is a technological evolution of broadcast television and advancement over analog television[1]. DTTV broadcasts land-based (terrestrial) signals. The advantages of digital terrestrial television, are similar to digital versus analog in platforms such as cable, satellite, and all telecommunications; the efficient use of spectrum and provision of more capacity than analog, better quality images, and lower operating costs for broadcast and transmission (after the initial upgrade costs). A terrestrial implementation of digital television (DTV) technology uses an aerial to broadcast to a conventional television antenna (or aerial) instead of a satellite dish or cable television connection[2].

Competing variants of broadcast television systems are being used around the world.

i) Advanced Television Standards Committee created the ATSC standards that use an ATSC tuner in North America and South Korea-an evolution from the analog National Television System Committee (NTSC) standard.

ii) Integrated Services Digital Broadcasting (ISDB$\mathrm{T}$ ) is used in Japan, with a variation of it being used in most of South America.

iii) DVB-T is the most prevalent, covering Europe, Australia, New Zealand, Colombia and some countries of Africa.

This paper proposes a way by which the existing Digital Video Broadcasting- Terrestrial (DVB-T) bandwidth may be modified so that the Internet content may be allocated space is looked at. Proper Conditional Access (CA)

DOI 10.17148/IARJSET.2015.2210 content, Internet content or both.

With standards for satellite TV, cable TV, and DVB-T already existing in Europe, America and the rest of the world [3], to come up with this system, strict guideline has to be followed to make sure that the already squeezed DVB-T bandwidth is not interfered with. Mathematical models may be developed or the improvement of existing models may be done to ensure compatibility with existing systems [4]. We analyse the bandwidth and how it can be shared by both the TV signal and Internet data.

\section{II.CURRENT TRENDS}

Existing DVB-T standards can be divided into two major categories as: Single Carrier (SC) and Multi Carrier (MC) or Orthogonal Frequency Division Multiplexing (OFDM). OFDM has been successful technique in noisy environment which are severely affected by multipath fading. OFDM divides the high frequency carrier into a group of subcarriers which are parallel to each other. Each sub-carrier is modulated and orthogonally packed using the Inverse Fourier Fast Fourier Transform (IFFT). Line of sight (LOS) cannot be guaranteed in digital TV, precaution has to be taken to avoid Inter-Symbol Interference (ISI) and fading. OFDM is affected by flat fading, which can be reduced by one tap equalisation. One tap equalization is achieved by compensation from only one tap delay.

\section{Transmission}

DTTV is transmitted on radio frequencies through terrestrial space in the same way as standard analog television, with the primary difference being the use of multiplex transmitters to allow reception of multiple channels on a single frequency range (such as a UHF or VHF channel) known as sub-channels.

Reception:

DTTV is received either via a digital set-top box (STB), TV gateway or integrated tuner included with television 
sets, that decodes the signal received via a standard accommodate the increased data rate. 64-QAM is the basic television antenna. Some set-top-boxes and TV gateways platform used in digital systems . If 256-QAM modulation include digital video recorder (DVR) functionality.

\section{III.MODULATION AND CODING} scheme is used, extra parallel bits are added which helps to carry the Internet data. Since Terrestrial digital TV is designed to lower data rates through compression using This paper increases the segments in ISDB-T to lossy techniques, while the Internet and broadcast data can accommodate the Internet payload. However data rate is be compression further using non-lossy techniques. Figure affected, a better way of digital modulation is chosen to 1 shows the basic system format.

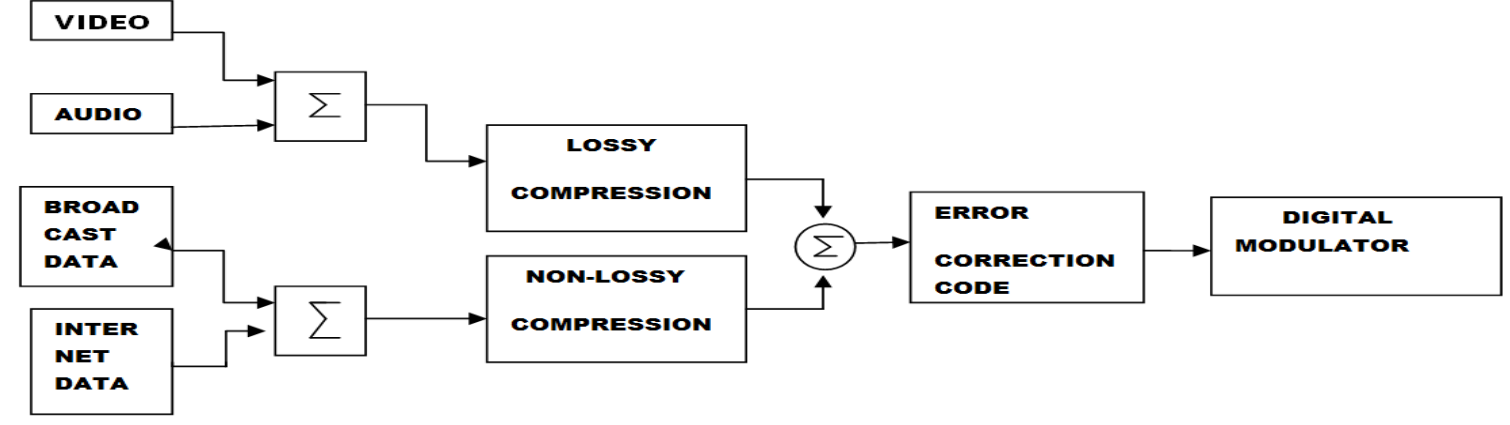

Fig: 1 Modulator Block Diagram

For better performance in multi-fading environment, the best case scenario will be, a very long GI with shortest system should adopt OFDM. The ISDB-T adopts a 13 guard fraction, this of course will achieve excellent segment OFDM to accommodate terrestrial video and a multipath performance and a higher capacity in the single segment for mobile reception. Modulation channel.

techniques used for digital broadcasting include QPSK, If a smaller guard interval is selected the DVB-T system 16-QAM, 32-QAM and even 64-QAM. QPSK is a would achieve a better transmission efficiency. It should common modulation technique employed in satellite be noted however that the increase in the value of $\mathrm{M}$ in Mcommunication. The requirement here is that the ary modulation scheme does not affect the number of bandwidth be sufficient enough to accommodate both OFDM carriers, but in a multipath fading environment like audio, video, broadcast data and Internet data. It should be in DVB systems increase in carrier is recommended as this noted that the number of carriers used will not affect the will limit the effect of echoes during transmission. The band bandwidth however it is the modulation technique choice of inner coding is selected to allow more and the Guard Interval (GI) that determine the bandwidth information bits per code. The challenge is that while that the broadcaster and the Internet Service Provider more data is carried compromise is made on protection. (ISP) may use. Another way of accommodating more data The reduction in the number of codeword bits mean especially that of the Internet is to use Multiple Physical reduced redundancy. The ISDB-T has the lowest code rate Layer Pipes (MPLP) that can maintain standard at $7 / 8$ so a recommendation is made for even lower code modulation for broadcast video, audio and data but a rate of up to and above 10/11. In ISDB-T the transmission different modulation for Internet data. When there is need of TV signal consists of 13 successive OFDM segments to increase the capacity of a DTTB channel the GI is taken blocks which are also known as segments, the bandwidth in serious consideration because increasing it improves the of each segment is equal to $1 / 14$ of the TV broadcasting system performance against multipath fading but reduces channel[5]. In a basic form ISDB-T is implemented as the system capacity. Ts $=\Delta+\mathrm{Tu}$ Where $\Delta=$ Guard Interval. illustrated in Figure 2. Where is shows how Transmission $\mathrm{Tu}=$ active carrier interval Ts=the total system interval A Streams (TS) are re-multiplexed to form a main TS that is later channel coded

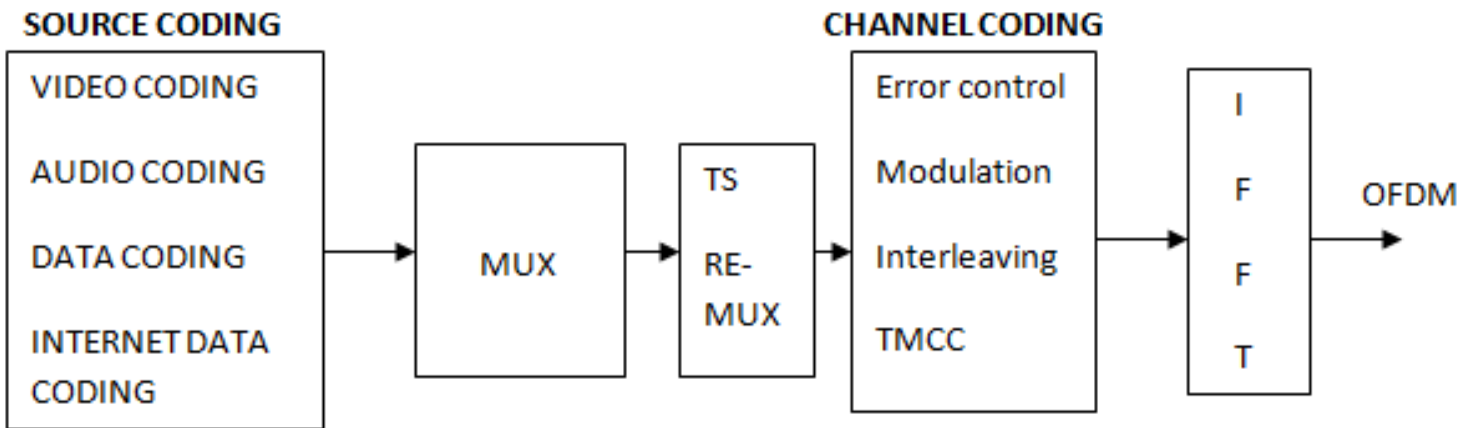

Figure.2 Improved ISDB-T DTTB system 
The high demands for quality picture and audio put a lot of emphasis on the amount of data to be transmitted. Currently most DTTB use MPEG-4 and lately MPEG-H for video and MP3 quality audio. For the broadcast data and the Internet data it would be appropriate to use both lossless and lossy compression technique where possible. Optimized Register Transfer Logic (RTL) design and implementation of LZW algorithm for high bandwidth applications [6] would be a better choice for the Internet data proposed in this paper. The optimized RTL design provides a comparatively high throughput $1.42 \mathrm{Gbits} / \mathrm{s}$, elevated throughput/slice value $151.8 \mathrm{Kbytes} / \mathrm{s} / \mathrm{slice}$ and lower operational power requirements $333 \mathrm{~mW}$. Over the ISO/IEC 23008-2 MPEG-H series of standards for video compression the High Efficiency Video Coding (HEVC) is a recommendation.

\section{IV.SET-TOP BOX CONFIGURATION}

The design of the set-top box is recommended to have an RJ45 port for a patch cord Personal Computer (PC) connection. The Figure 3.0 shows a home setup for the set-top for PC and TV. Terrestrial set-top boxes are meant to use the limited network broadcast network signal from a local service provider. Common set-top boxes are meant to receive and decode digital signal to for analog TV input. The set-top box proposed here will have two outputs for both TV and a computer capable of receiving data signals through the RJ45 network adapter. The internet line can be connected to a router, useful for wired and wireless purposes.

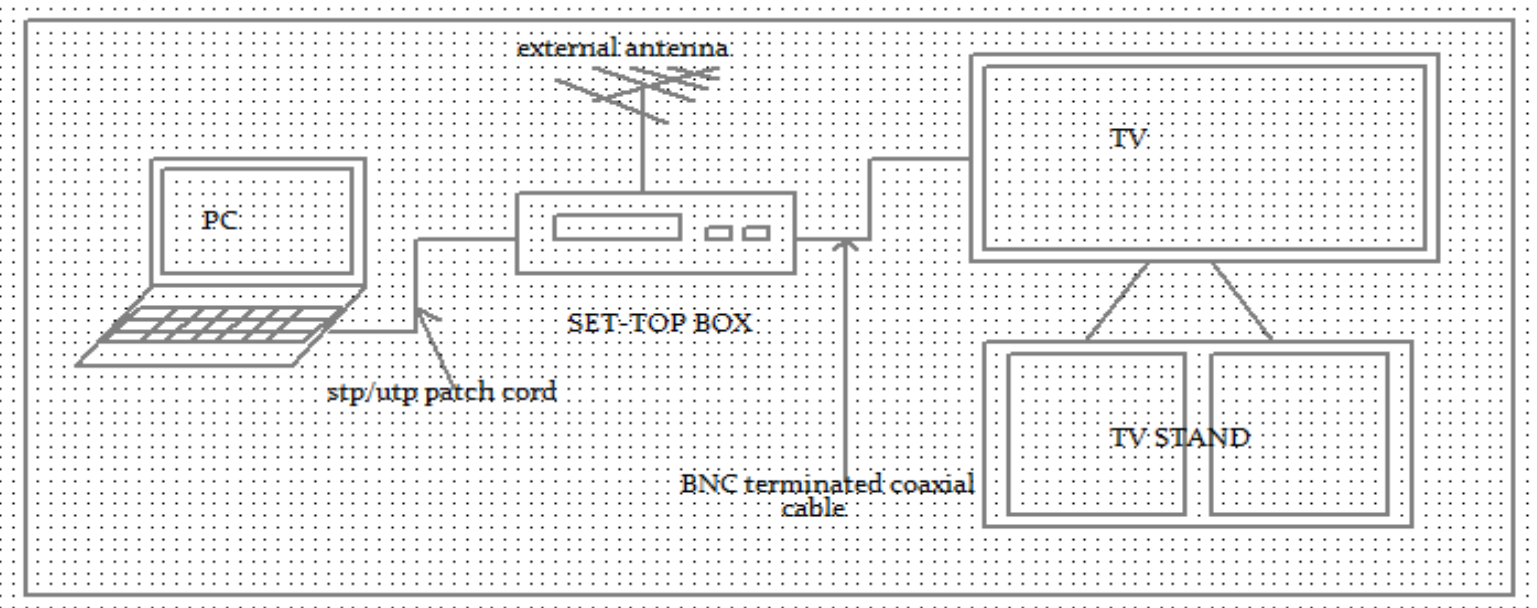

\section{V.CONCLUSION}

Figure 3 Set-top box home configuration

This paper has only proposed one method among the many methods that DVB-T can be used to improve user experience by allowing the user to connect and access the Internet through there set-top boxes. Yet there are many techniques that DVB-T system can be used to improve bandwidth, compression, source coding and line coding. And also when the proposed idea comes into implementation, there may be design constraints that need to overcome. Other issues which may as well be considered in further research include the modification of the existing set-top boxes software for Conditional Access (CA).

\section{REFERENCES}

[1]. O'Leary, Seamus "Understanding Digital Terrestrial Broadcasting",Artech House Digital Audio and Video Library" ISBN 1-58053-063-X

[2]. Beutler, Roland "Digital Terrestrial Broadcasting Networks" 2009, ISBN 978-0-387-09634-6, Vol. 23

[3]. Ana García Armada and Miguel Calvo Ramón "Rapid Prototyping Of A Test Modem For Terrestrial Broadcasting Of Digital Television" IEEE Transactions on consumer electronics, vol. 43, no. 4 (Nov. 1997), pp. 1100-1109

[4]. Kazuo Takayama, Kohichi Chikaishi, Toshio Tanaka, Hidenori Gohhara "Development of Terrestrial TV Broadcast" Fujitsu Ten Technical Journal No. 272006

[5]. „Transmission System for Digital Terrestrial TV Broadcasting” ARIB STD-B31 v 1.62005.

[6]. Navqi, Saud; Naqvi, R.; Riaz, R.A.; Siddiqui, F. (April 2011). "Optimized RTL design and implementation of LZW algorithm for high bandwidth applications". Electrical Review 2011 (4): 279-285 